\title{
Application of Vibrational Spectroscopy in Biology and Medicine. Breath Analysis ${ }^{+}$
}

\author{
Alexander Apolonski ${ }^{1,2}$, Susmita Roy ${ }^{3}$, Renée Lampe ${ }^{3,4}$ and Kiran Sankar Maiti ${ }^{1,2, *}$ \\ 1 Max Planck Institute of Quantum Optics, Hans-Kopfermann Straße 1, D-85748 Garching, Germany; \\ Alexander.Apolonskiy@mpq.mpg.de \\ 2 Ludwig Maximilian University Munich, Chair of Experimental Physics - Laser Physics, Faculty of Physics, Am \\ Coulombwall 1, D-85748 Garching, Germany \\ 3 Research Unit of the Buhl-Strohmaier Foundation for Cerebral Palsy and Paediatric Neuroorthopaedics, \\ Klinikum rechts der Isar, Technical University of Munich, D-81675, Munich, Germany; susmita.roy@tum.de \\ (S.R.); renee.lampe@tum.de (R.L.) \\ 4 Markus Würth Professorship, Technical University of Munich, D-81675 Munich, Germany \\ * Correspondence: kiran.maiti@mpq.mpg.de; Tel.: +49-89-289-14112 \\ + Presented at the 15th International Workshop on Advanced Infrared Technology and Applications (AITA 2019), \\ Florence, Italy, 17-19 September 2019.
}

Published: 23 September 2019

\begin{abstract}
Vibrational spectroscopy is a powerful technique to study the molecular structure and dynamics. It has a great promise to use for medical diagnosis. It is already proved that volatile organic compound contain in breath, reflect the internal chemistry of the body. However, due to the lack of reliable investigation technique, identification and quantification of those molecules are not reached to clinically acceptable parameter. Fourier transform infrared (FTIR) based vibrational spectroscopy has been used to investigate the breath sample. Molecular identification based component analysis of breath sample is proposed.
\end{abstract}

Keywords: Vibrational spectroscopy; volatile organic components (VOC); component analysis

\section{Introduction}

Vibrational spectroscopy is well established as a powerful and widely used technique for the study of molecular structure and dynamics in the fields of chemistry and physics [1]. It uses molecular bond vibration as a probe to identify the molecule and their structure [2]. In recent years, development of short pulse ultrafast laser makes it possible to observe the vibrational motion in real time [3]. This unique feature facilitate to reveal chemical and biological processes even in femtosecond (fs) timescale [4]. Therefore, it is natural to think that vibrational spectroscopy can be a potential diagnosis method in medical science [5].

Vibrational spectroscopy uses infrared light to excite molecular bonds and measure absorption and transmission of light due to the vibrations of the bonds. Each chemical bond has an unique vibrational energy [6,7]. Due to this unique vibrational energy, each molecule will have an unique spectral features. This unique spectral feature is called fingerprint of the molecule. The molecular fingerprinting is used to identify a particular molecule from a mixture, therefore, it is very important for biological and medical sample analysis.

Vibrational spectroscopy is not only efficient to identify molecules from mixture, but also it has two advantages over other existing techniques for biological sample analysis. (a) The reagent-free analysis of 
biofluids takes advantage of the fact that a multitude of analytes can be quantified simultaneously. (b) Since the infrared beam only make changes on the vibrational level, molecules remain intact. Therefore, there is no chance of damage of tissue or constituent molecules in biological sample [8]. However, due to high absorption of infrared radiation by water, penetration depth in the tissue is very small, which limits the use of this technique for medical diagnosis.

It has been already proved that small volatile organic components (VOC) escaping from body by means of breath, body odor, etc., reflect the internal chemistry of the body [9]. They are not only characteristic of a person, but also specific to different kinds of diseases. VOCs, related to diseases could serve as biomarkers for clinical diagnostics and disease monitoring. Among many, breath is a source of large number of VOCs and easy to collect for diagnosis. A significant effort has been devoted for the analysis of the VOCs, using Mass spectroscopy, electric nose, vibrational spectroscopy, etc., however, each technique has their inherent shortcoming. Breath contain a large amount of water vapor, which is the main obstacle for the vibrational spectroscopy. Due to the lack of appropriate water removal technique, vibrational spectroscopy until recently not a popular technique for breath analysis.

The main advantage of breath analysis using infrared spectroscopy over the tissue or liquid phase biological sample is, water can be removed from the sample without changing the constitute molecules. A highly efficient water removal technique has been developed and reported recently [10]. In combination with water removal technique, Fourier transform infrared (FTIR) spectroscopy already proved its potential to breath analysis [11]. In this article, it has been shown, how FTIR spectroscopy is used for analysis of breath samples.

\section{Results and Discussions}

For a large number of samples, it is a common practice that medical data are typically analysed statistically. Most commonly used statistical methods are principal component analysis (PCA), analysis of variance (ANOVA), random forest, etc. In all these cases, data are analysed blindly, therefore, any measurement errors or noise, affect the statistical data analysis, which leads to misinterpretation of the data. We propose component analysis method, where each of the spectral component is matched with molecular fingerprint, in case of match, spectral component is considered for analysis. Since only identified spectral features are consider for analysis, there is no chance of misinterpretation of the data. The strength of the spectral peak is used to determine the state of the body [11]. A detail analysis of the body state is out of scope of this article, however, the method of component analysis is explained below in detail.

A typical water suppressed breath spectra of a healthy volunteer is presented in Figure 1. A large number of spectral features are observed in the spectra. In general without water suppression most of them are buried under water spectra except carbon dioxide $\left(\mathrm{CO}_{2}\right)$. It is produced in many biological processes in our body, however, it is not informative enough, because, it is difficult, even impossible to quantify the amount is produced by each individual processes. On the other hand, many small VOCs in breath, are produced in a single biological processes, specially in case of disease. The main task of breath analysis is to identify those particular VOCs and finding out their origin in the body. 


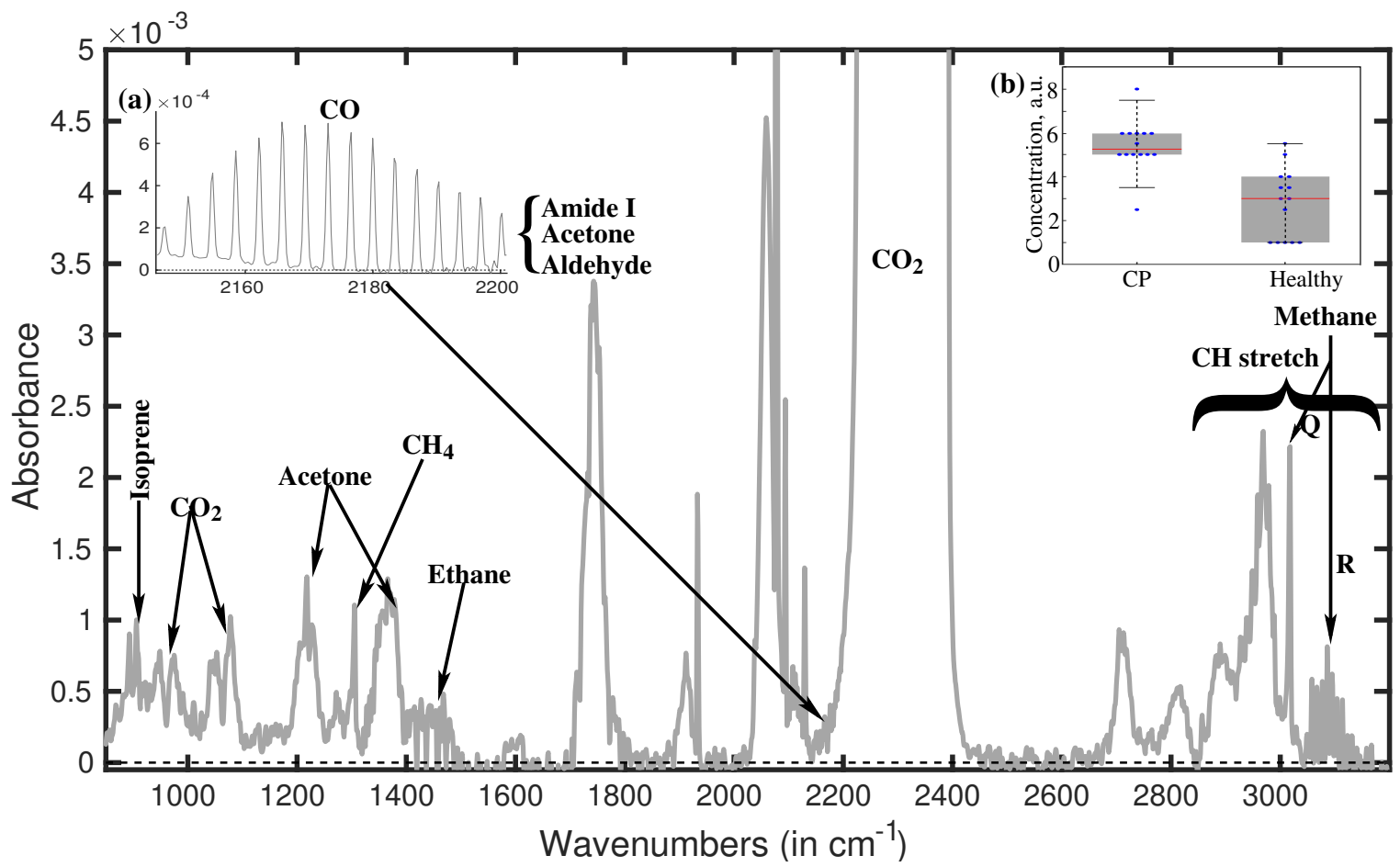

Figure 1. Water suppressed breath spectra of a healthy volunteer. In inset (b) spectral strength of peak at $1189 \mathrm{~cm}^{-1}$ Few fingerprints are labeled. Many fingerprints are still buried under these strong peaks.

The spectral range $\left(2800-3200 \mathrm{~cm}^{-1}\right)$ in general populated with $\mathrm{C}-\mathrm{H}$ stretch vibrational absorptions [12]. The $\mathrm{CH}$ bond is the characteristics of the biological molecules, therefore all biological molecules yield their $\mathrm{CH}$ absorption peaks in this spectral region, and appear as very congested spectral feature. Therefore, practically it is impossible to identify individual molecule with their fingerprints in the $\mathrm{CH}$ stretch vibrational region. However, a distinguish spectral feature around $3100 \mathrm{~cm}^{-1}$ is identified as $\mathbf{R}$ branch of Methane. Very strong $\mathbf{Q}$ branch is observed at $3020 \mathrm{~cm}^{-1}$ but $\mathbf{P}$ branch of methane is buried under the broad $\mathrm{C}-\mathrm{H}$ absorption spectra of other molecules.

Many prominent spectral features are observed in between $900-1800 \mathrm{~cm}^{-1}$. The spectral features in this spectral region are unique to individual molecules and relatively less congested, which makes it easier to identify the molecules using molecular fingerprint. For example, double peaks at around $900 \mathrm{~cm}^{-1}$ is an unique feature for Isoprene. Double peaks at $893 \mathrm{~cm}^{-1}$ and $906 \mathrm{~cm}^{-1}$, indicate the presence of Isoprene in the breath sample. The peak at $1220 \mathrm{~cm}^{-1}$ is a clear signature of Acetone and identified unambiguously. However, the peak at $1375 \mathrm{~cm}^{-1}$, which also a fingerprint of Acetone, is much higher in strength than it should be. Therefore, this spectral feature must be formed due to absorption of more than one molecule. We have identified the second molecule, Acetaldehyde. A very strong absorption is observed at $1750 \mathrm{~cm}^{-1}$ due to the absorption of the $\mathrm{C}=\mathrm{O}$ of acetone, acetaldehyde and some small molecule with amide band.

The identified molecules, mentioned above, are present in breath of healthy individual with relatively high concentration, between few hundred ppb (parts-per-billion) to few ppm (parts-per-million). However, many more molecules are present in breath with much lower concentration, even down to ppt (parts-per-trillion) level. In the current state, limits of our detection system is about $50 \mathrm{ppb}$. Therefore, there is a chance to find out more molecules in the spectra. With a proper magnification of the spectra, many more molecules can be found on the pedestal of the above mentioned peaks. For example, a fine structure is visible (see inset of Figure1(a)) at the left pedestal of $\mathrm{CO}_{2}$, by magnifying the spectra ten times. This fine structure is identified as carbon monoxide, which in general produce in the body due to the 
oxidative stress and tissue injury. Similarly using higher magnification and careful investigation many more molecules can be identified. So far we have identified 14 VOCs, however many more distinct spectral features are on the way for identification.

Using above mentioned component analysis, a pilot study has been performed with 14 cerebral palsy volunteers and 14 healthy volunteers. Two strong distinguish spectral features have been observed at $1189 \mathrm{~cm}^{-1}$ (I) and $1203 \mathrm{~cm}^{-1}$ (II), between two group of volunteers. Both the spectral features are with similar spectral width of $10 \mathrm{~cm}^{-1}$. The signatures found for $100 \%$ (I) and $86 \%$ (II) of the CP patients and present for $92 \%$ (I) and $83 \%$ (II) of healthy individuals, are in average higher in concentration for the former cohort by approximately a factor of two, without any individual exception. The strength of the peak at $1189 \mathrm{~cm}^{-1}$ for two groups of volunteers are presented at inset of Figure1(b). By performing a statistical analysis based on above mentioned two spectral feature we have found better than $90 \%$ accuracy in distinguishing the individuals in the groups.

\section{Materials and Methods}

Fourteen persons with CP (23-52 years), recruited from a day care center for persons with disabilities and 14 healthy adults (30-77years), recruited among the personnel working at the University were participated in this study. For the participants with $\mathrm{CP}$, the experimental procedures were approved by the ethics committee of the Faculty of Medicine of the Technical University of Munich and for the healthy participants from the ethics committee of the Ludwig-Maximilians-University. Participation in the study was voluntary, and all subjects, or in some cases their legal protectors, gave their written informed consent before collecting the samples. Each breath sample was collected in a single use 1 liter Tedlar bag. The sample was passed through the water suppression machine before collecting to sample cell for measurement. After the water suppression, the dried gas sample came into a $4 \mathrm{~m}$ multipass cell sitting in Bruker Vertex70 spectrometer. For data analysis, we performed manual inspection of spectral features, with the correction of the baseline. Earlier, we found that blind principal component analysis is not reliable for the purposes of high-quality comparison of the cohorts [11]. The data analysis has been performed by matching the fingerprint of the molecules.

\section{Conclusions}

Infrared based breath analysis method has been discussed in the article. FTIR spectroscopy has been used to record the absorption spectra of water suppressed breath samples. The molecular fingerprint based spectral analysis is used to identify the VOCs in exhaled breath. So far 14 VOCs have been identified. There are many more absorption peaks still need to be identify. A pilot study has been performed with 14 cerebral palsy and 14 healthy volunteers. Using component analysis of breath spectra, better than $90 \%$ accuracy has been achieved, to identify the two groups.

Author Contributions: K.S.M. and A.A. triggered and coordinated the investigation, K.S.M. made literature search and K.S.M and S.R prepared the manuscript, K.S.M. built the experimental setup, performed the experiments and data analysis, R.L., A.A. and K.S.M. arranged the volunteer group, S.R., R.L. and K.S.M. collected the samples.

Acknowledgments: We acknowledge financial support from DFG via 'Munich Center for Advanced Photonics'. The authors thank the therapists and participants of the MFZ for their time and support to this study.

\section{References}

1. Ultrafast Infrared Vibrational Spectroscopy, 1st ed.; Fayer, M.D., Ed.; CRC Press: New York, NY, USA; London, UK, 2013; ISBN 9781466510135

2. Maiti, K.S. Vibrational spectroscopy of Methyl benzoate. Phys. Chem. Chem. Phys. 2015, 17, 19735-19744, doi:10.1039/C5CP02281A 
3. Hochstrasser, R.M. Two-dimensional spectroscopy at infrared and optical frequencies. Proc. Natl. Acad. Sci. USA 2007, 104, 14190-14196, doi:10.1073/pnas.0704079104

4. Maiti, K.S. Broadband two dimensional infrared spectroscopy of cyclic peptide 2-Pyrrolidinone. Phys. Chem. Chem. Phys. 2015, 17, 24998-25003, doi:10.1039/C5CP04272K

5. Heise, H.M. Medical Applications of Infrared Spectroscopy. In Progress in Fourier Transform Spectroscopy; Mink, J., Keresztury, G., Kellner, R., Eds.; Springer: Vienna, Austria, 1997; pp. 67-77.

6. Maiti, K.S.; Scheurer, C. Basis set extrapolation for high resolution spectroscopy. J. Chem. Chem. Eng. 2013, 7, 1100-1110, doi:10.17265/1934-7375/2013.11.014.

7. Maiti, K.S. Ultrafast N-H vibrational dynamics of hydrogen-bonded cyclic amide reveal by 2DIR spectroscopy. Chem. Phys. 2018, 515, 509-512, doi:10.1016/j.chemphys.2018.10.003

8. Pahlow, S.; Weber, K.; Popp, J.; Wood, B.R.; Kochan, K.; Rüther, A.; Perez-Guaita, D.; Heraud, P.; Stone, N.; Dudgeon, A.; et al. Application of Vibrational Spectroscopy and Imaging to Point-of-Care Medicine: A Review. Appl. Spectrosc. 2018, 72, 52-84, doi:10.1177/0003702818791939.

9. Volatile Biomarker. Non-Invasive Diagnosis in Physiology and Medicine; Amann, A., Smith, D., Eds.; Elsevier, 2013; eBook ISBN: 9780444626202, Hardcover ISBN: 9780444626134

10. Maiti, K.S.; Lewton, M.; Fill, E.; Apolonski, A. Sensitive spectroscopic breath analysis by water condensation. J. Breath Res. 2018, 12, 046003, doi:10.1088/1752-7163/aad207

11. Maiti, K. S.; Lewton, M.; Fill, E.; Apolonski, A. Human beings as islands of stability. Breath study. Sci. Rep. 2019, under review.

12. Roy, S.; Maiti, K.S. Structural sensitivity of C-H vibrational band in Methyl benzoate. Spectrochim. Acta Mol. Biomol. Spectrosc. 2018, 196, 289, doi:10.1016/j.saa.2018.02.031

(C) 2019 by the authors. Licensee MDPI, Basel, Switzerland. This article is an open access article distributed under the terms and conditions of the Creative Commons Attribution (CC BY) license (http:/ / creativecommons.org/licenses/by/4.0/). 